\title{
Die Versorgung von Mukoviszidose-Patienten
}

\author{
Behandlung, Screening-Programme und klinische Outcomes
}

\author{
Birgitta Strandvik
}

Department of Pediatrics, Institute of Clinical Sciences, Sahlgrenska Academy, Göteborg University, Göteborg, Sweden

\section{Schlüsselwörter \\ Ernährung • Essenzielle Fettsäuren · Staphylococcus aureus • Pseudomonas aeruginosa $\cdot$ Intravenöse Behandlung zu Hause $\cdot$ Transplantation $\cdot$ Neugeborenen-Screening • Überlebensrate}

\section{Zusammenfassung}

Die in der Pädiatrie häufigste autosomale Erbrankheit, die Mukoviszidose (cystische Fibrose, CF), ist inzwischen nicht mehr auf die Kindheit beschränkt, da die modernen Behandlungsmöglichkeiten den von dieser Erkrankung betroffenen Patienten sowohl eine längere Überlebensdauer als auch eine bessere Lebensqualität beschert haben. Als das Gen vor über 16 Jahren identifiziert und die häufigste Mutation, $\Delta F 508$, festgestellt wurde, hoffte man allgemein, bald eine Heilung für diese Erkrankung zu finden. Mit dieser Entdeckung nahmen die Aktivitäten zur Erforschung der Pathophysiologie der Erkrankung stark zu, aber bis heute sind die bei der klinischen Behandlung erzielten Fortschritte gänzlich auf Verbesserungen der traditionellen Behandlung zurückzuführen. Der verbesserte Zustand der Patienten hängt mit einer verbesserten Substitution der Pankreasenzyme und einer intensiveren Ernährungstherapie zusammen, einschliesslich der Aufmerksamkeit, die dem gestörten Lipidstoffwechsel geschenkt wird. Ermöglicht durch die Verfügbarkeit einer Reihe wirksamer Antibiotika und die Entwicklung neuer Behandlungsstrategien kann die Lungenfunktion inzwischen mittels Behandlung der pulmonalen Erkrankung bis ins Erwachsenenalter erhalten werden. Diese Strategien umfassen schleimlösende Mittel, Bewegungsförderung von klein auf in Kombination mit einer veränderten Physiotherapie sowie bessere Ergebnisse bei der Lungentransplantation als letzte Möglichkeit der Behandlung. Lebererkrankungen werden inzwischen besser erkannt und Ursodeoxylcholsäure häufiger zur langfristigen Behandlung eingesetzt, selbst wenn ihre Wirkung hinsichtlich der Prävention einer weiteren Progression der Lebererkrankung noch nicht vollständig geklärt ist. Das Neugeborenen-Screening nimmt zu und bringt einerseits kurzfristige Probleme mit sich, z.B. psychische Probleme, wenn Träger oder sehr milde Formen der Erkrankung diagnostiziert werden, dient aber andererseits der Erforschung langfristiger Ergebnisse, mittels derer belegt werden kann, dass sich der klinische Zustand bessert und die Überlebensdauer weiter steigt. Die Tatsache, dass die Überlebensdauer nach wie vor begrenzt ist, scheint in erster Linie auf die nach wie vor rätselhafte chronische Kolonisierung der Atemwege durch Mikroorganismen zurückzuführen zu sein. C Copyright $\odot 2006$ Nestec Ltd., Vevey/S. Karger AG, Basel

\section{Einleitung}

Als die cystische Fibrose (CF) 1936-1938 als eigenständige, von der Zöliakie unabhängige Erkrankung identifiziert wurde $[1,2]$, war die Behandlung noch sehr beschränkt und die Mortalität in den ersten Lebens-

\section{KARGER \\ Fax +4161306 1234 \\ E-Mail karger@karger.ch}

www.karger.com (c) 2006 Nestec Ltd., Vevey/S. Karger AG, Basel 0250-9652/06/0643-0133\$23.50/0

Online-Zugriff auf: www.karger.com/and
Prof. Birgitta Strandvik

Department of Pediatrics, Queen Silvia Children's Hospital

SE-41685 Göteborg (Sweden)

Tel. +46 31343 4723, Fax +46 31217023

E-Mail Birgitta.Strandvik@pediat.gu.se 
jahren daher hoch [3]. Obwohl bereits 1960 über den ersten Patienten berichtet wurde, bei dem die Diagnose der Erkrankung im Erwachsenenalter erfolgte [4], ist das Wissen über diese Erkrankung in der Ärzteschaft nach wie vor häufig unzureichend, so dass milde Fälle möglicherweise ein Leben lang unerkannt bleiben. Ein Problem bei der Behandlung der CF-Patienten besteht darin, dass sich sehr milde Fälle nach einer Virusinfektion oder aufgrund anderer, bisher unerkannter Faktoren auch rapide verschlechtern können, ein Umstand, der darauf hindeutet, dass es bei der Diagnose CF wichtig ist, auch milde Symptome zu behandeln. Dies spricht für ein Neugeborenen-Screening, selbst wenn die Krankheit bisher nicht heilbar ist. Da die Wirksamkeit der Enzymsubstitution zu Anfang nicht zufriedenstellend war und massive Ernährungsprobleme vorlagen, überwog die Mortalität im Kindesalter, und die Versorgung von CF-Patienten oblag hauptsächlich den pädiatrischen Gastroenterologen. Mit Entwicklung der magensaftresistenten Mikrotabletten zur Substitution der Pankreasenzyme verbesserte sich die Wirksamkeit der Enzymersatztherapie und führte zu einer besseren Nährstoffabsorption und somit einer besseren Überlebensrate in den ersten Lebensjahren, so dass die pulmonalen Symptome stärker beachtet wurden. Dank der grossen Zahl neuer $\beta$-Laktam-Antibiotika konnte man sich in den 70er Jahren des 20. Jahrhunderts stärker auf die Behandlung der pulmonalen Erkrankung konzentrieren und je mehr die Therapie der Lungenerkrankung verbessert wurde, desto stärker fiel die Versorgung der CF-Patienten in den Verantwortungsbereich der Lungenfachärzte. Da sich inzwischen die Ergebnisse der Lungentransplantation zunehmend verbessern, wird sich die Versorgung schwerpunktmässig wieder den gastrointestinalen Komplikationen, wie Leber- und Gallenerkrankungen und anderen mit der CF verbundenen Problemen, z.B. Nierenerkrankungen, Knochenbrüchigkeit, Diabetes und Fertilitätsproblemen, zuwenden. Dies wiederum wird die Notwendigkeit einer zentralen, von einem Expertenteam ausgeführten Versorgung von CF-Patienten aufzeigen. Je länger die Überlebensdauer von CF-Patienten und je besser ihre Voraussetzungen auf ein normales Leben in der Gesellschaft, desto mehr bedarf es einer Anpassung der Behandlungsstrategien, um eine bessere Compliance $\mathrm{zu}$ erzielen. Dies wird die Experten hinsichtlich der medizinischen Versorgung und Pflege von CF-Patienten vor neue Herausforderungen stellen.

\section{Organisation der Versorgung von CF-Patienten}

Bereits in den 60er Jahren des 20. Jahrhunderts hat man erkannt, dass die Pflege und Versorgung von CFPatienten in medizinischen Zentren zu einer längeren Überlebensdauer führte und auch die enge Zusammenarbeit zwischen den CF-Zentren und den an den CFProblemen interessierten Ärzten vor Ort zu einer besseren Pflege und Versorgung beitragen kann, wenn sich die Patienten mindestens einmal jährlich in dem für sie zuständigen CF-Zentrum untersuchen lassen [5]. Der Grund hierfür ist, dass praktizierende Ärzte über ein hohes Mass an Erfahrung verfügen müssen, um die feinen Unterschiede der zutage tretenden Symptome erkennen und eine optimale Behandlung gewährleisten zu können. Daraus ergibt sich auch, dass ein auf die Behandlung von CF-Patienten spezialisiertes Zentrum nicht zu klein (d.h. dass dort mindestens 50 Patienten behandelt werden sollten), aber wahrscheinlich auch nicht zu gross sein sollte, da die richtige Einschätzung des aktuellen Krankheitszustands eines Patienten und einer möglicherweise notwendigen Intensivierung der Behandlung leichter ist, wenn man den Patienten persönlich kennt. Es hat sich gezeigt, dass sich das Überleben und der Allgemeinzustand von Patienten verbessern, wenn diese mindestens einmal monatlich zur Untersuchung kommen. Inzwischen weiss man auch, dass in einem Mukoviszidose-Zentrum ein Expertenteam bestehend aus einem Arzt, einer Krankenschwester oder einem Pfleger, einem Physiotherapeuten, einem Ernährungsexperten, einem Sozialpädagogen, einem Psychologen, einem Schriftführer und einem technischen Assistenten für den Schweisstest bereit stehen sollte [6]. Ferner besteht Bedarf an Spezialdienstleistungen, z.B. die Zusammenarbeit mit Genetikern, einer bakteriologischen Einrichtung, die Interesse an den spezifischen Problemen hat, Kulturen vom Sputum von CF-Patienten zu züchten, einer klinisch-physiologischen Abteilung, die daran interessiert ist, die pulmonale Erkrankung zu bewerten, einer Röntgeneinrichtung, etc. Einige Mukoviszidose-Zentren sollten eng mit medizinischen Einrichtungen, die Lungen- und Lebertransplantationen vornehmen, zusammenarbeiten.

\section{Energiebedarf}

CF-Patienten haben einen höheren Stoffwechsel und ihre Energiezufuhr sollte daher 120\% im Vergleich zu einer gesunden Kontrollperson betragen [7]. Ihr Gesamt- 
energieverbrauch ist je nach körperlicher Aktivität und Einschränkung der Lungenfunktion unterschiedlich, die Ergebnisse bezüglich eines Zusammenhangs mit dem Genotyp sind jedoch widersprüchlich $[8,9]$. Einige Patienten gedeihen auch ohne zusätzliche Energiesupplementierung [10], aber die meisten Studien belegen, dass schon die Einhaltung der empfohlenen Energiezufuhr für CF-Patienten im Allgemeinen schwierig ist, und dies trägt möglicherweise dazu bei, dass CF-Patienten schwer ein normales Wachstum erzielen [11]. In diesem Zusammenhang ist zu erwähnen, dass Patienten mit Mutationen, die angeblich mit einem hohen Energiebedarf verbunden sind, wie z.B. $\Delta$ F508, auch eine Pankreasinsuffizienz aufweisen und anscheinend anfälliger für einen Mangel an essenziellen Fettsäuren sind [12]. Ein Mangel an essenziellen Fettsäuren wird bei Ratten mit einem höheren Energieverbrauch in Verbindung gebracht.

Um eine hohe Energiezufuhr zu erzielen, müssen diese Patienten sich energiereich ernähren, d.h. eine Diät zu sich nehmen, bei der 40-45\% der Energie aus Fett gewonnen wird (E\%), während bei der normalen Ernährung eines gesunden Erwachsenen maximal 30\% der Energie aus Fett stammt; eine derart hohe Fettzufuhr erreichen jedoch nur wenige Patienten [11]. Es gilt jedoch, die hinreichend bekannten niedrigen Linolsäurekonzentrationen im Blutplasma (18:2n-6) zu korrigieren. 10-15 E\% sollten daher mit ungesättigten Fettsäuren abgedeckt werden. Mittels einer hohen Zufuhr an Omega-6-Fettsäuren lassen sich in der Regel die niedrigen Serumspiegel an Docosahexaensäure normalisieren [12, 13]. Die CF-Patienten profitieren von einer erhöhten Aufnahme an Omega-3-Fettsäuren, da die Eicosanoide der Eicosapentaensäure (20:5n-3), das Prostaglandin $\mathrm{E}_{3}$ und die Leukotriene der 5er-Reihe entzündungshemmend sind und der erhöhten Eicosanoidproduktion aus der Arachidonsäurekaskade (20:4n-6) entgegenwirken, die bei CFPatienten erhöht ist $[14,15]$ und die meisten Entzündungen stimuliert.

Durch das mit der modernen Mukoviszidose-Behandlung einhergehende erhöhte Mass an Sport und Bewegung steigt der Energiebedarf. Die pulmonale Verschlechterung geht mit einer erhöhten Atemfrequenz und mit den allgemeinen Infektionsfolgen einher und führt insbesondere bei schwer kranken Patienten zu einer weiteren Steigerung des Energiebedarfs. Gleichzeitig wirkt der durch eine Infektion hervorgerufene Zytokinanstieg möglicherweise appetithemmend, ein Umstand, der die Nahrungsaufnahme bei CF-Patienten häufig erschwert [16]. Hier ist Findigkeit gefragt, um die Patienten zum
Essen anzuregen und so eine Verlagerung in Richtung eines katabolischen Stoffwechsels zu vermeiden, der sich schnell zu einem Zustand entwickeln kann, in dem invasive Massnahmen, wie z.B. enterale Ernährung, notwendig werden.

Die im Handel erhältlichen Nahrungsergänzungsmittel können als Zwischenmahlzeiten zur Steigerung der Energiezufuhr eingesetzt werden, eignen sich aber nicht als Ersatz für die Hauptmahlzeiten, da sie einen relativ hohen Kohlenhydratgehalt haben und ihre mittelkettige Fettsäureketten (mittelkettige Triglyceride) keinen Ersatz für Linolsäure darstellen. Eine Verschlechterung der Atemwegsinfektionen kann bei Kleinkindern möglicherweise zu einer vorübergehenden Beeinträchtigung der Restfunktion der Bauchspeicheldrüse führen und Diarrhöe bzw. gastroenterologische Beschwerden sind möglicherweise die ersten Symptome, welche die Notwendigkeit einer aggressiveren Behandlung der Pankreasinsuffizienz und der pulmonalen Infektion anzeigen.

Inzwischen gibt es zwar sowohl bei stabilem Zustand als auch während Exazerbationen eine Enzymersatztherapie, aber die Normalisierung der Fettabsorption erweist sich trotzdem häufig als schwierig, ein Umstand, der darauf hinweist, dass diese Therapie weiter verbessert werden sollte und dass weitere Faktoren an der gestörten Fettabsorption beteiligt sind [17]. Klinische Studien untersuchen derzeit neue Enzymersatztherapien, von bakteriellen Lipasen bis zu der rekombinant hergestellten, durch humanes Gallensalz-stimulierten Lipase [18, 19]. Eine schwere Lebererkrankung tritt selten auf und es ist somit unwahrscheinlich, dass eine verminderte Gallensäuresynthese eine wichtige Rolle bei der gestörten Lipolyse spielt [20]. Gallenkomplikationen können jedoch den Gallenfluss senken und somit zu niedrigen intraluminalen Konzentrationen von Gallensalz und zur Malabsorption beitragen [21]. Ein weiterer möglicherweise wichtiger Faktor ist, dass die auf einen Mangel an essenziellen Fettsäuren hinweisende, niedrige Linolsäurekonzentration im Blutplasma eventuell den transzellulären Fetttransport senkt und so zu einer Störung der Fettabsorption führt [22]. Aufgrund der gestörten Absorption essenzieller Fettsäuren, d.h. aufgrund der niedrigen Linolsäurekonzentrationen, könnte bei einem schweren Mangel möglicherweise sogar eine intravenöse Verabreichung von Linolsäure notwendig werden [23]. Patienten mit Mutationen, die mit einem schweren Phänotyp assoziiert werden, sind anfälliger für niedrigere Linolsäurekonzentrationen im Blutplasma und brauchen möglicherweise regelmässigere Supplementationen [12]. Unter 
praktischen Gesichtspunkten ist es möglicherweise empfehlenswert, während der letzten 3 Tage einer aufgrund einer pulmonalen Exazerbation durchgeführten Antibiose den intravenösen Zugang zu nutzen und täglich über 6-8 Stunden $10 \mathrm{ml}$ Intralipid 10\% (oder die entsprechende Menge einer vergleichbaren Fettemulsion)/kg Körpergewicht intravenös zu verabreichen. Wichtig ist hierbei, dass die Infusion nicht zu schnell und auch nicht in der akuten Phase der Infektion erfolgen darf, da die Aktivität der Lipoproteinlipase möglicherweise herabgesetzt ist. Spezialvorrichtungen zur Infusion von Fettemulsionen sind hier vorzuziehen. Bei Patienten mit einem schweren Mangel an essenziellen Fettsäuren kann 4 bis 6 Monate lang alle 2 bis 3 Wochen eine ähnliche dreitägige Behandlung empfohlen werden, um die Linolsäurekonzentrationen im Blutserum $\mathrm{zu}$ normalisieren und dadurch die Nieren- und Leberfunktionen zu verbessern $[24,25]$.

Bei Patienten, bei denen bereits eine Mangelernährung in Verbindung mit einer stark beeinträchtigten Lungenfunktion vorliegt, ist möglicherweise eine enterale Ernährung (Nasen-Magen-Sonde oder Gastroenterostomie) notwendig, um eine normale Körpergrösse und ein normales Körpergewicht zu erreichen [26]. Eine enterale Nahrung mit hoher Energiedichte $(1 \mathrm{kcal} / \mathrm{ml})$ kann vorzugsweise nachts verabreicht werden, um störende Auswirkungen auf die normale Nahrungsaufnahme bzw. den normalen Tagesablauf zu vermeiden. Enzymsupplementierung und der Start der Infusion vor dem Schlafengehen reichen in der Regel bereits für eine zufriedenstellende Absorption. Diese Form der Behandlung ist häufig bei Patienten erforderlich, die auf eine Transplantation warten, noch effizienter ist sie jedoch unter Umständen, wenn sie bei Problemen mit der Nahrungsaufnahme frühzeitig und in diesen Fällen möglicherweise auch nur vorübergehend zum Einsatz kommt. Eine totale parenterale Ernährung ist in der Regel nicht indiziert.

\section{Vitaminsupplementierung}

Aufgrund der Schwierigkeit, die Fettabsorption zu normalisieren, bedarf es einer zusätzlichen Supplementierung mit fettlöslichen Vitaminen. Da die oxidative Kapazität bei CF-Patienten aus unbekannten Gründen erhöht ist [27], muss den Antioxidanzien, wie z.B. Vitamin $\mathrm{E}$ und den Beta-Karotinen, deren Konzentrationen bei CF-Patienten in der Regel sehr niedrig sind, besondere Aufmerksamkeit zuteil werden. Vitamin A ist für die mukosale Integrität wichtig, bedarf aber nach einer Transplantation, nach der die Konzentrationen im Blutserum häufig sehr stark ansteigen, der besonderen Auf- merksamkeit. Die Serumkonzentrationen der fettlöslichen Vitamine müssen regelmässig, mindestens jährlich, überwacht werden, insbesondere angesichts der Bedenken einer möglichen Toxizität hoher Konzentrationen. Einen Überblick verschaffen die jüngsten 'Consensus'-Berichte, die eine Zusammenfassung der Ernährungspraktiken enthalten $[28,29]$. Tabelle 1 enthält eine Zusammenfassung der Ernährungsempfehlungen bei Mukoviszidose.

\section{Umgang mit Darmproblemen}

In manchen Fällen hängt Appetitlosigkeit mit wiederkehrenden Bauchschmerzen, aufgrund von Darmproblemen, wie z.B. einer Stenose oder dem distalen intestinalen Obstruktionssyndrom (DIOS), zusammen. Während bei einer Stenose ein chirurgischer Eingriff erforderlich ist, kann das distale intestinale Obstruktionssyndrom in der Regel erfolgreich mit Hilfe eines Einlaufs mit $10 \%$ N-Acetylcystein und hyperosmolarem Röntgenkontrastmittel im Verhältnis von 1:1 oder 2:1 behandelt werden. Die verabreichte Menge an $N$-Acetylcystein sollte maximal $300 \mathrm{mg} / \mathrm{kg}$ Körpergewicht betragen. Bei Patienten mit häufig rezidivierenden Symptomen kann die tägliche Einnahme grosser Mengen inerter Polymere, wie z.B. eine Mischung von Polyethylenglykol verschiedener Grössen, in Kombination mit einer Ernährungsumstellung, bei der ballaststoffreiche Nahrungsmittel vermieden werden, eine gewisse Erleichterung verschaffen [30].

\section{Behandlung von Lungenerkrankungen}

Der Behandlung von Atemwegssymptomen wurde bislang die meiste Aufmerksamkeit zuteil, da die Lungenerkrankung für 95\% der Mortalität bei CF verantwortlich zeichnet. Zwar beruhen alle Behandlungsstrategien auf einer Kombination aus schleimlösenden Mitteln, passiver oder aktiver Physiotherapie und Antibiose, aber bezüglich des Erfolgs sind zwischen den einzelnen Ländern und Zentren grosse Unterschiede festzustellen [31]. Die meiste Aufmerksamkeit wurde der chronischen Kolonisierung mit Pseudomonas aeruginosa zuteil, obwohl die, dieser in der Regel vorausgehende, mehr oder weniger chronische Kolonisierung mit Staphylococcus aureus einen viel stärkeren sprunghaften Anstieg des Sauerstoffverbrauchs ('respiratory burst') zur Folge hat und daher auch viel aggressiver auf das Gewebe wirkt [32]. Es besteht das allgemeine Risiko, dass die Behandlung des S. aureus-Befalls aus Furcht vor einem Pseudo- 
Tabelle 1. Empfohlene tägliche Nährstoffaufnahme bei Mukoviszidose ${ }^{1}$

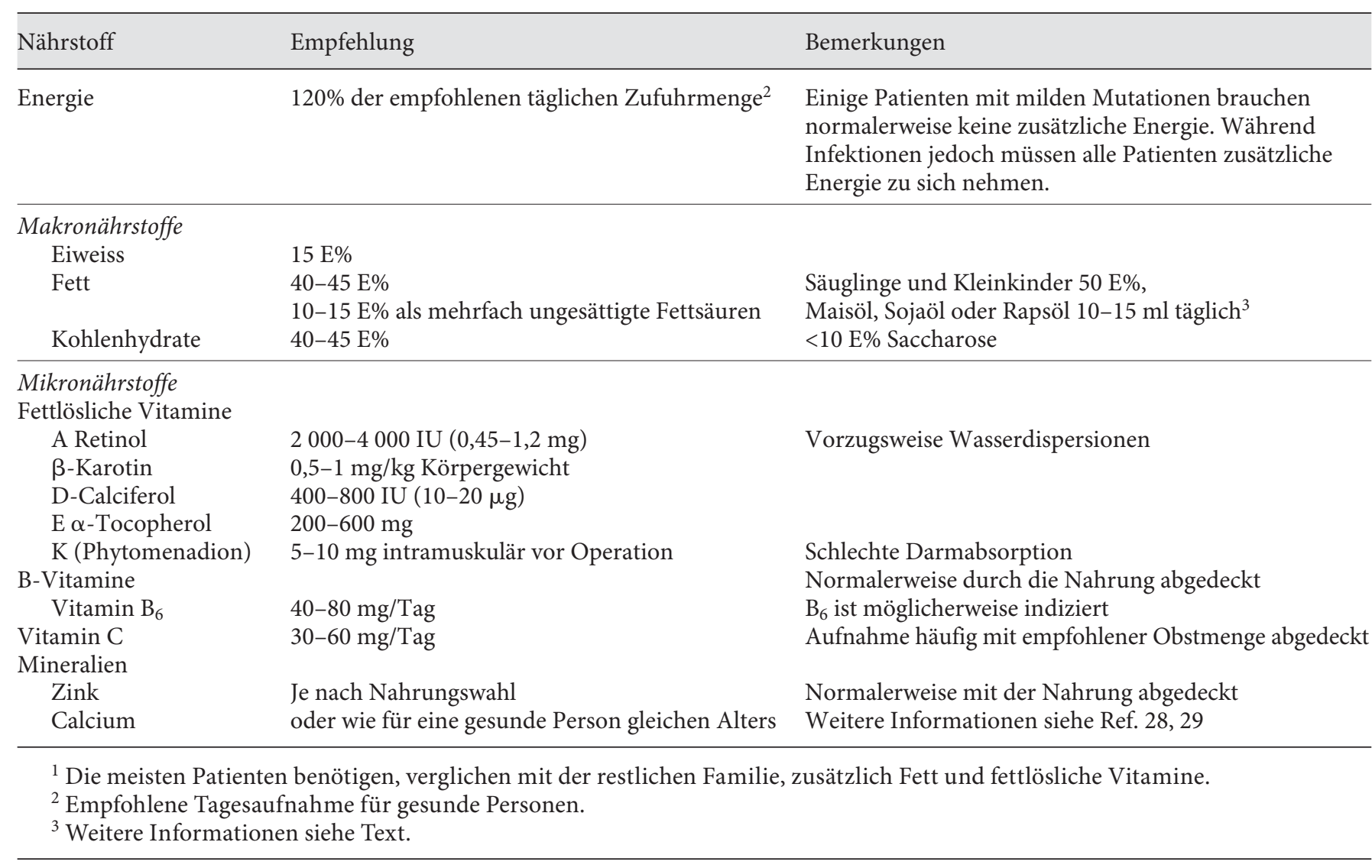

monas-Befall, der eine intravenöse Antibiotikabehandlung erforderlich macht, unbewusst vernachlässigt wird. Pseudomonas-Spezies sind in der Natur weit verbreitete, fakultativ aerobe Bakterien. Werden Patienten bei Pseudomonas-Befall bereits bei milden Symptomen aggressiv behandelt, so können sie diese jahrzehntelang ohne Verschlechterung der Lungenfunktion in sich tragen [33]. Weitere bei CF häufig auftretende Bakterien sind Haemophilus influenzae und Stenotrophomonas maltophilia. Unter der Bezeichnung Burkholderia cepacia wird eine grosse Anzahl Bakterien zusammengefasst, von denen einige Stämme mit einer schweren und ansteckenden Lungeninfektion in Verbindung gebracht werden, die häufig als 'Cepacia-Syndrom' bezeichnet wird.

Die in vielen CF-Zentren entwickelten intensiven und langfristigen Antibiosen haben Probleme mit resistenten Stämmen mit sich gebracht. Da man eine Übertragung dieser Stämme zwischen den Patienten fürchtet, werden die Patienten nach der Art ihrer bakteriellen Kolonisierung getrennt, was für die Patienten eine grosse psychische Belastung mit sich bringt $[34,35]$. In einem

Die Versorgung von

Mukoviszidose-Patienten
Cochrane-Bericht jüngeren Datums wurde die Frage aufgeworfen, ob sich die hohen Antibiotikamengen angesichts der Probleme mit resistenten Stämmen und der Trennung der Patienten tatsächlich dazu eignen, zu vermeiden, dass sich eine Kolonisierung mit Pseudomonas zu einer chronischen Infektion entwickelt [36]. Ein Übersichtsartikel jüngeren Datums, der sich mit den Behandlungsstrategien in Skandinavien befasst, zeigt, dass es dort Zentren gibt, in denen diese hohen Antibiotikamengen nicht verabreicht werden. In diesen Zentren ist die Pseudomonas-Prävalenz bei den Patienten zwar vergleichsweise höher, gleichzeitig aber treten weniger Probleme mit resistenten Stämmen auf [37]. Körpergrösse, Körpergewicht und Lungenfunktion der in diesen Zentren behandelten Patienten sind gut und dies wirkt sich auch auf die Lebensqualität dieser Patienten aus, ein Umstand, der darauf hindeutet, dass alternative Behandlungsstrategien durchaus in Betracht gezogen werden sollten [Lindblad A, et al., in einer künftigen Veröffentlichung des skandinavischen CF-Konsortiums] (Abb. 1).

Ann Nestlé [Ger] 2006;64:133-144 
Tabelle 2. Die häufigsten Behandlungen der pulmonalen Beschwerden mit Mukolytika und Antibiotika ${ }^{1}$

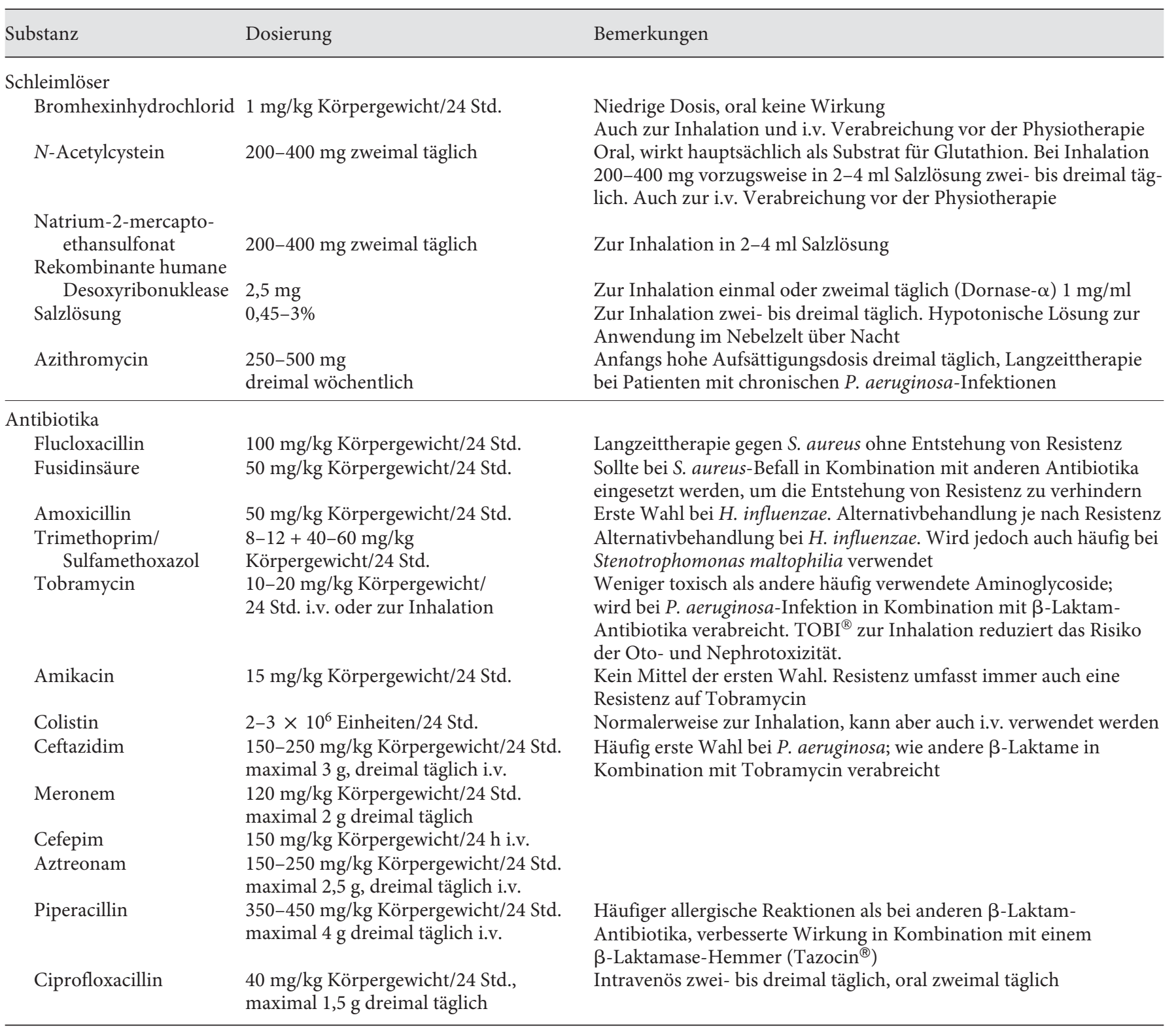

${ }^{1}$ Mukolytika und Antibiotika werden häufig im Kombination eingesetzt, wobei jedes Zentrum seine eigene Strategie verfolgt.

Tabelle 2 enthält eine Auflistung diverser Agenzien, die häufig zur Behandlung der Lungenerkrankung eingesetzt werden. Es wurden verschiedene Behandlungsstrategien entwickelt, die sich in der Mehrzahl darauf konzentrieren, eine chronische Kolonisierung mit grammnegativen Bakterien, insbesondere P. aerugino$s a$, zu verhindern. Die Behandlungsstrategien umfassen sowohl allgemeine schleimlösende Mittel, Physiotherapie wie auch Antibiose. Der sehr klebrige Schleim haftet an der Schleimhaut und trägt als Substrat zur bakteriellen Kolonisierung bei (Abb. 2). Lautet die Diagnose CF, so leiten die meisten Zentren, um diese Entwicklung zu vermeiden, eine Monobehandlung mit schleimlösenden Agenzien ein, bis die Bakterien nachweislich isoliert sind. Körperliche Bewegung kann Bestandteil dieser Behandlungsstrategie sein [38] und umfasst möglicherweise eine von klein auf mit Hilfe von Bällen und Trampolinen durchgeführte Physiotherapie. In einigen Län- 


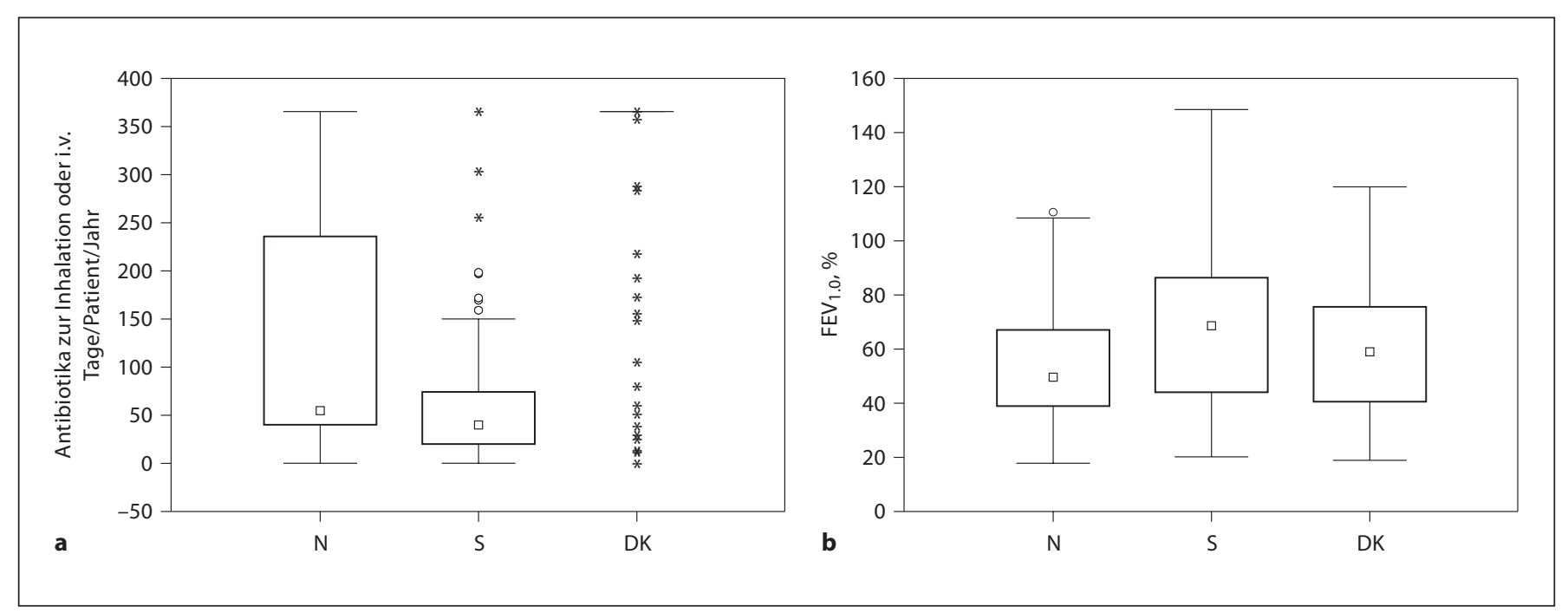

Abb. 1. Box-and-Whisker-Plots der Behandlung von Pseudomonas-Infektionen bei Patienten im Alter von über 19 Jahren mit chronischem $P$. aeruginosa-Befall in den drei skandinavischen Ländern Norwegen (N), Schweden (S) und Dänemark (DK; a) sowie der Lungenfunktion dieser Patienten ausgedrückt als forciertes expiratorisches Volumen in $1 \mathrm{~s}\left(\mathrm{FEV}_{1.0}\right)$ als Prozentsatz der Sollwerte (b). a Zahl der Tage pro Jahr, an denen die Patienten

dern dominiert nach wie vor eine passivere Form der Physiotherapie, bei der die Patienten täglich unter der Anleitung eines Physiotherapeuten mit den Händen klatschen. Lautet die Diagnose CF, so erhält der Patient ausserdem oral Bromhexin verabreicht, um zu verhindern, dass es durch die Ansammlung von klebrigem Schleim zur Ausbildung eines Schleimpfropfens kommt, und um die Symptome einer chronischen Sinusitis zu mildern.

Auch die tägliche Inhalation mit Salzlösung, Broncholytika und Schleimlösern wird früh eingeleitet; die Frequenz richtet sich in der Regel nach den Symptomen. Alle diese Arzneimittel fördern bei Patienten mit pulmonalen Symptomen nachweislich die Expektoration und die Lungenfunktion; welche Rolle sie im Rahmen der Prophylaxe spielen, ist jedoch nicht geklärt. Unter klinischen Gesichtspunkten sollte die Wahl des Arzneimittels auf den einzelnen Patienten abgestimmt werden, d.h. bei manchen Patienten wird die beste Wirkung mit Dornase- $\alpha$ erreicht, während bei anderen bereits mit Salzlösung eine ebenso gute Wirkung erzielt wird $[39,40]$. Die Schleimexpektoration wird bei allen Patienten am besten mittels Inhalationstherapie kombiniert mit Sport bzw. Physiotherapie erzielt, wobei letztere, um eine optimale Compliance zu erzielen, ebenfalls individuell auf die $\mathrm{Pa}$ - mittels Inhalation oder i.v. mit Antibiotika behandelt wurden. Die Ergebnisdarstellung zeigt den Median (25. und 75. Perzentile). Die Whisker stellen die 5. und 95. Perzentilen dar, die Punkte zeigen die Ausreisser an und Extremwerte werden als Sternchen dargestellt. Veröffentlichung mit freundlicher Genehmigung des skandinavischen CF-Konsortiums, Dr. A. Lindblad.

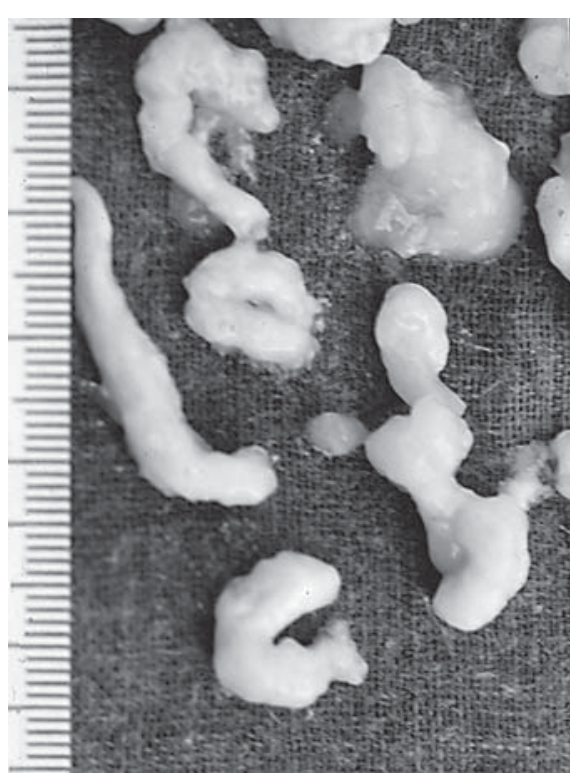

Abb. 2. Schleimpfropfen, die mittels Bronchoskopie bei einem Kind mit cystischer Fibrose entfernt wurden. Photo: mit freundlicher Genehmigung von Dr. Jean Feigelson, Paris. 
tienten zugeschnitten sein sollten. In den letzten Jahren wurde Azithromycin als Mukolytikum eingesetzt und zeigte bei Patienten mit chronischen Pseudomonas-Infektionen eine bemerkenswerte Wirkung, obwohl die Bakterien nicht empfindlich gegenüber dem Wirkstoff sind. Durch Langzeitbehandlung mit diesem Mukolytikum gelang es, die Anzahl der intravenösen Antibiosen zu verringern.

\section{Behandlungsstrategien mit Antibiotika}

Im Allgemeinen wird eine Infektion mit $S$. aureus behandelt, sobald das Pathogen isoliert wurde und die Wirkung der Behandlung sich leicht durch spezifische Antikörper im Blutserum, wie z.B. Antikörper gegen Teichonsäure und $\alpha$-Toxine, überwachen lässt [41]. Da Sputumproben bei Kleinkindern möglicherweise nur schwer zu gewinnen sind, wird in einigen CF-Zentren bei Kleinkindern eine Langzeitprophylaxe mit Fusidinsäure oder Flucloxacillin durchgeführt, während andere Zentren aus Angst, eine solche Behandlung könne die Kolonisierung mit grammnegativen Bakterien beschleunigen, von einer solchen Langzeitprophylaxe absehen. Die Anmerkung, dass eine Resistenz gegenüber Flucloxacillin extrem selten ist und dass in Ländern, wie z.B. Schweden, in denen dieses Arzneimittel zur Langzeitbehandlung eingesetzt wird, Methicillin-resistente Stämme nach wie vor sehr selten isoliert werden, ist in diesem Zusammenhang möglicherweise wichtig [37].

Derzeit wird bei der ersten Isolation von $P$. aeruginosa in der Regel sofort aggressiv behandelt und die chronische Kolonisierung so hinausgezögert. In den meisten Zentren kommt eine Antibiotika-Therapie zum Einsatz, bei der zuerst eine Kombination aus Aminoglykosiden, normalerweise Tobramycin, und einem $\beta$-Laktam-Antibiotikum intravenös verabreicht wird, gefolgt von einer wochen- oder sogar monatelangen Inhalationstherapie mit Tobramycin oder Colistin [42]. Einige Zentren führen eine regelmässige Inhalationstherapie mit Tobramycin $\left(\mathrm{TOBI}^{\circledR}\right)$ durch, bei der auf eine vierwöchige Behandlung eine vierwöchige Behandlungspause folgt, während andere Zentren auf ein ähnliches Regime der Inhalationsbehandlung allerdings mit Colistin bauen. Je intensiver und länger die jeweilige Behandlung, desto geringer das Risiko eines chronischen Befalls (der wie folgt definiert wird: mehr als eine Isolation von $P$. aeruginosa in einem Zeitraum von 6 Monaten und positive Pseudomonas-Antikörpertiter [43]); stattdessen wird für eine intermittierende Kolonisierung der Preis eines hohen Antibiotikakonsums in Kauf genommen. Diese intensive Antibiose führt ferner zu einer chronischen Kolonisierung mit anderen Bakterienarten, z.B. dem Achromobacter xylosoxidans. Wie aus Abbildung 1 ersichtlich, war die Lungenfunktion der Patienten in dem skandinavischen Land mit dem geringsten Antibiotikumkonsum mit der Lungenfunktion der Patienten aus den anderen Ländern vergleichbar, ein Umstand, der darauf hindeutet, dass die Art der Behandlungsstrategien bei der Antibiose von grosser Wichtigkeit ist. Bei einigen schwerkranken Patienten mit nur schlecht auf Antibiotika ansprechenden Pseudomonas-Stämmen wurden mittels Dauerinfusion mit $\beta$-Laktamen gute Wirkungen erzielt. Das Risiko, eine Resistenz zu entwickeln, ist im Allgemeinen geringer, wenn die Behandlung auf 7-14 (durchschnittlich 10) Tage beschränkt wird. Erweist sich eine längere Therapie als erforderlich, so sollte das Antibiotikum gewechselt werden [33-37]. Wir haben die Erfahrung gemacht, dass die Behandlung mukoider Stämme einfacher ist als die Behandlung nicht-mukoider Stämme. Warum dies so ist, wissen wir bisher nicht, aber möglicherweise besteht ein Zusammenhang mit der Behandlungsstrategie, bei der Mukolytika und Antibiotika zusammen eingesetzt werden.

Die am weitesten verbreitete Behandlungsmodalität ist, bei einer sich abzeichnenden Exazerbation der pulmonalen Symptome eine intravenöse Antibiose einzuleiten. In dem Kampf, die Zahl der infektiösen Erreger so niedrig wie möglich zu halten, hat man in den letzten Jahren häufiger auf eine mehr oder minder durchgängige Inhalationstherapie zurückgegriffen. Mit Entwicklung eines speziell für die Inhalationstherapie vorgesehenen Tobramycinpräparats (TOBI p) haben sich die Behandlungsgrundsätze in vielen Ländern verändert, weg von einer intravenösen und hin zu einer langfristigen Inhalationstherapie, bei der auf eine vierwöchige Behandlung eine vierwöchige Behandlungspause folgt und für die der Nachweis erbracht wurde, dass sie die Lungenfunktion verbessert und über längere Zeiträume ziemlich konstant hält [44]. Ähnliche Ergebnisse lassen sich mit einer konventionelleren Kombinationstherapie mit Tobramycin und $\beta$-Laktamen erzielen, bei der ferner weniger Probleme mit einer antimikrobiellen Resistenz der bakteriellen Stämme entstehen [33, 45]. Dies zeigt sich auch anhand der Tatsache, dass in dem skandinavischen Land, mit dem bei CF-Patienten niedrigsten Antibiotikakonsum, die Inhalationstherapie nur selten eingesetzt wird. Die Kosten für die intravenöse Therapie lassen sich ebenfalls relativ gering halten, indem man Eltern und Patienten lehrt, wie die intravenöse Antibiose zu Hause durchgeführt werden kann, und dadurch den Familien mehr Freiräume verschafft, was wiederum eine bessere 
Compliance und eine höhere Akzeptanz der Behandlung zur Folge hat [46]. Auch die Blutproben, die bei jeder i.v. Antibiose nach der dritten Dosis mit Aminoglykosiden erforderlich sind, da die Dosis zur Vermeidung einer eventuellen Toxizität kontrolliert und eventuell angepasst werden muss, können zu Hause abgenommen und dann an das Krankenhaus geliefert werden.

Ein Problem entsteht manchmal, wenn es gegenüber dem antimikrobiellen Wirkstoff der Wahl oder sogar dem einzigen Wirkstoff, gegenüber dem in vitro eine Empfindlichkeit der Erreger festgestellt wurde, zu einer allergischen Reaktion kommt. Dieses Problem lässt sich durch eine sorgfältige Desensibilisierung lösen, bei der man die Behandlung mit sehr niedrigen Konzentrationen des Antibiotikums einschleicht [47]. Längere Behandlungszeiträume können insbesondere bei schwer kranken Patienten, die auf eine Lungentransplantation warten, erforderlich sein und in diesen Fällen ist ein Wechsel des Antibiotikums wichtig.

\section{Pro und Kontra Steroidbehandlung}

Steroide werden manchmal zur Entzündungsbekämpfung verwendet, steigern jedoch bei systemischer Verabreichung das Risiko eines klinischen Diabetes und sollten daher nur restriktiv eingesetzt werden. Zwar hat der Einsatz neuer, inhalativ wirksamer Formulierungen zugenommen, aber bisher wurde noch in keiner Studie nachgewiesen, dass eine Steroidbehandlung eine bessere antiinflammatorische Wirkung zeigt als eine Antibiose [48].

\section{Behandlung der Hypoxämie}

Die periphere Oximetrie sollte regelmässig überprüft werden, da sich die Oxygenierung auch bei anscheinend gut erhaltener Lungenfunktion schleichend verschlechtern kann. Die dieser Entwicklung zugrunde liegenden Mechanismen sind nicht klar. Die ersten Anzeichen entwickeln sich normalerweise nachts bei pulmonalen Exazerbationen [49], und obwohl dies hinreichend bekannt ist, gibt es bislang keine CF-Studien, die zeigen, wie dieses Problem am besten behandelt werden könnte [50]. Patienten mit einer moderaten Störung der Oxygenierung wird oft eine nächtliche Sauerstoffsupplementation empfohlen, aber ab einem bestimmten Grad der Störung ist eine kontinuierliche Sauerstoffversorgung notwendig. Bei Patienten, die auf eine Transplantation warten, kann sich die nicht-invasive Beatmung als hilfreich erweisen, die auch bei schweren Exazerbationen eine notwendige Ergänzung der Behandlung darstellen kann [51]. Eine Beatmungshilfe mittels Beatmungsgerät ist kontraindi- ziert, da der Patient möglicherweise nicht extubiert werden kann.

\section{Indikation für eine Lungentransplantation}

Bei Patienten mit einer Lungenfunktion von nur noch $\mathrm{FEV}_{1.0}<30 \%$ der Sollwerte sollte eine Transplantation in Betracht gezogen werden. Bei der Entscheidung, den Patienten auf eine Warteliste zur Lungentransplantation $\mathrm{zu}$ setzen, gilt es, viele Faktoren zu berücksichtigen, z.B. Ernährung, Geschwindigkeit der Abnahme der Lungenfunktion, Art der bakteriellen Kolonisierung, psychische Faktoren, etc. [52].

\section{Früherkennung}

Die CF-Früherkennung wurde erstmals in den $60 \mathrm{er}$ Jahren des 20. Jahrhunderts eingeführt, wobei die Albuminkonzentrationen im Stuhl als ein Indikator für Pankreasinsuffizienz dienten. Aufgrund grösserer Probleme mit sowohl falsch positiven als auch falsch negativen Ergebnissen hat sich diese Methode jedoch nie richtig durchgesetzt. Man fand heraus, dass ein erhöhter Gehalt an immunreaktivem Trypsin eine höhere Spezifizität und Sensitivität für die Früherkennung der Mukoviszidose hat, und daher wird dieser Laborparameter seit kurzem als erstes bestimmt, die Bestimmung in manchen Fällen wiederholt und im Anschluss daran in der Regel eine Mutationsanalyse der Mutationen mit der in der jeweiligen Region höchsten Prävalenz vorgenommen, bevor ein Schweisstest durchgeführt wird [53]. Mit der Einführung einer Früherkennung soll die Entwicklung eines Lungenschadens vermieden werden und kurzfristig werden sowohl hinsichtlich des Ernährungszustands als auch hinsichtlich der Lungenerkrankung positive Ergebnisse erzielt [54]; ab einem Alter von 10 Jahren jedoch sind die Unterschiede zwischen den Patienten mit und den Patienten ohne Früherkennung in den meisten Studien äusserst gering [55]. Dies ist enttäuschend und der jüngste Consensus-Bericht spricht nach wie vor keine Empfehlung für eine allgemeine Früherkennung aus, hält sie jedoch in bestimmten Regionen und für spezielle Zwecke für angebracht [53]. Dank vieler sorgfältiger Follow-ups bei Patienten, bei denen eine Früherkennung durchgeführt wurde, wissen wir inzwischen mehr über den natürlichen Verlauf der frühen Manifestationen dieser Erkrankung. Zu den Problemen, die sich in Folge einer Früherkennung ergeben, zählen die Diagnose von Fällen ohne bzw. mit sehr schwach ausgeprägten Frühsymptomen, die Erkennung von Trägern, Störungen in 
der frühen Mutter-Kind-Bindung, die Tatsache, dass schwach ausgeprägte Symptome bei Patienten, die in der Früherkennung übersehen wurden, nicht erkannt werden, sowie die Kosten [55]. Da die CF bisher nicht geheilt werden kann, sollte eine regionale Einführung der Früherkennung in enger Zusammenarbeit mit den CF-Zentren erwogen werden und die Kosten und Nutzen sowohl für jeden einzelnen Patienten als auch für die Gesellschaft als Ganzes ausgewogen sein.

\section{Outcome}

Die CF ist nicht länger eine auf die Pädiatrie beschränkte Krankheit. In immer mehr Zentren sind die Hälfte oder annähernd die Hälfte der behandelten Patienten Erwachsene. Die mittlere Überlebensdauer ist gestiegen und beträgt sowohl in den USA als auch in Westeuropa mindestens 25 Jahre, in einigen Zentren sogar sehr viel mehr [56]. In den anderen Ländern gibt es jedoch, meist aufgrund sozioökonomischer Faktoren, grosse regionale Unterschiede; in einigen Ländern des früheren Ostblocks beispielsweise stehen keine Behandlungsoptionen zur Verfügung, da selbst die medizinische Grundversorgung fehlt. In den Industrienationen können die meisten Patienten heutzutage ein erfülltes Sozialleben führen, Voll- oder Teilzeit arbeiten, und selbst die an CF erkrankten Männer können dank Spermienaspiration und nachfolgender In-vitro-Fertilisation eine Familie gründen. Zwar steigt mit zunehmendem Alter die Inzidenz des CF-Diabetes, aber dieser Diabetes-Typ ist in der Regel leicht zu behandeln und bei frühzeitiger Erkennung und Behandlung mit schnell wirkendem Insulin lässt sich die häufig in Zusammenhang mit dieser Komplikation gemeldete Verschlechterung des Patientenzustands verhindern. Eine Lebererkrankung wirkt sich praktisch nie auf das Überleben der Patienten aus. Erstens scheint das Alter normalerweise keinen Einfluss auf die Progression der Lebererkrankung zu haben und sollte dies doch einmal der Fall sein, so sind die inzwischen bei der Lebertransplantation erzielten Outcomes im All- gemeinen gut und gehen häufig sogar mit einer Verbesserung der Lungensymptome einher [57]. Eine kombinierte Lungen- und Lebertransplantation ist daher selten indiziert. Vor kurzem wurden jedoch kombinierte Transplantationen von Bauchspeicheldrüse und Leber erfolgreich durchgeführt. Eine weitere Verbesserung der Überlebensdauer wird dank der erfolgreichen Ergebnisse der Lungentransplantation erzielt. Bei CF-Patienten ist das 5-Jahres-Überleben im Allgemeinen, und insbesondere bei gutem Ernährungsstatus, besser als bei anderen Transplantationspatienten, wahrscheinlich weil sie jünger sind $[59,60]$.

\section{Schlussfolgerungen}

Der dieser Erkrankung zugrunde liegende Gendefekt wurde vor über 16 Jahren entdeckt und auf Grundlage dieser Entdeckung ist unser Wissen dank einer bemerkenswerten Zunahme der Grundlagenforschung merklich gewachsen. Es wurden mehrere potenzielle Innovationen für eine grundlegendere Behandlung der Erkrankung entwickelt, aber das stark verbesserte Überleben ist folgenden Faktoren zu verdanken: der kontinuierlichen Verbesserung der traditionellen Behandlung, der Versorgung der CF-Patienten in medizinischen Zentren und der Erkenntnis, dass eine häufige Kontrolle der Patienten dazu beitragen kann, auch schwach ausgeprägte pulmonale Symptome zu erkennen. Mittels einer aggressiven Behandlung wird das Entstehen progredient verlaufender Lungenschäden verhindert, wobei es dabei jedoch besonderer Aufmerksamkeit bedarf, um zu vermeiden, dass die langfristige Inhalationstherapie mit Antibiotika und die selteneren Besuche in der Klinik der Behandlungsintention entgegenwirken. Eine schwierige Aufgabe der medizinischen Zentren und der Behandlungsteams besteht darin, die Politiker dazu zu motivieren, die erforderlichen Mittel für die richtigen Behandlungen und Behandlungsoptionen, die im besten Interesse der Patienten sind, bereitzustellen.

\section{Literatur}

1 Fanconi G, Uehlinger E, Knauer C: Das Coeliakiesyndrom bei angeborenen zystischer Pancreasfibromatose und Bronchiektasien. Wien Med Wochenschr 1936;86:753-756.

2 Andersen DH: Cystic fibrosis of the pancreas and its relation to celiac disease: a clinical and pathological study. Am J Dis Child 1938; 56:344-399.
3 Bodian M: Fibrocystic Disease of the Pancreas: A Congenital Disorder of Mucus Production - Mucosis. New York, Grune \& Stratton, 1953.

4 Marks BL, Anderson CM: Fibrocystic disease of the pancreas in a man aged 46 . Lancet $1960 ; 1: 365$. 
5 van Koolwijk LME, Uiterwaal CSPM, van der Laag J, et al: Treatment of children with cystic fibrosis: central, local or both? Acta Paediatr 2002;91:972-977.

6 Littlewood JM: Good care for people with cystic fibrosis. Paediatr Respir Rev 2000;1: 179-189.

7 Thomson MA, Wilmott RW, Wainwright C, et al: Resting energy expenditure, pulmonary inflammation, and genotype in the early course of cystic fibrosis. J Pediatr 1996; 129:367-373.

8 O'Rawe A, McIntosh I, Dodge JA, et al: Increased energy expenditure in cystic fibrosis is associated with specific mutations. Clin Sci 1992;82:71-76.

9 Fried MD, Durie PR, Tsui L-C, et al: The cystic fibrosis gene and resting energy expenditure. J Pediatr 1991;119:913-916.

10 Kindstedt-Arfwidson K, Strandvik B: Food intake in patients with cystic fibrosis on an ordinary diet. Scand J Gastroenterol 1988; 23:160-162.

11 Kawchak DA, Zhao H, Scanlin TF, et al: Longitudinal, prospective analysis of dietary intake in children with cystic fibrosis. J Pediatr 1996;129:119-129.

12 Strandvik B, Gronowitz E, Enlund F, et al: Essential fatty acid deficiency in relation to genotype in patients with cystic fibrosis. J Pediatr 2001;139:650-655.

13 Farrell PM, Mischler EH, Engle MJ, et al: Fatty acid abnormalities in cystic fibrosis. Pediatr Res 1985;19:104-109.

14 Kurlandsky LE, Bennink MR, Webb PM, et al: The absorption and effect of dietary supplementation with omega- 3 fatty acids on serum leukotriene $\mathrm{B}_{4}$ in patients with cystic fibrosis. Pediatr Pulmonol 1994;18:211-217.

15 Strandvik B, Svensson E, Seyberth HW: Prostanoid biosynthesis in patients with cystic fibrosis. Prostagland Leukotr Essent Fatty Acids 1996;55:419-425.

16 Stark LJ: Can nutrition counselling be more behavioural? Lessons learned from dietary management of cystic fibrosis. Proc Nutr Soc 2003;62:793-799.

17 Baker SS, Borowitz D, Duffy L, et al: Pancreatic enzyme therapy and clinical outcomes in patients with cystic fibrosis. J Pediatr 2005;146:189-193.

18 Strandvik B, Hansson L, Hernell O, et al: Recombinant human bile salt-stimulated lipase improves lipid uptake and reduces the pancreatic enzyme supplementation in patients with cystic fibrosis. Pediatr Pulmonol 2004(suppl 27):333.

19 Borowitz D, Goss CH, Stevens C, et al: Safety and preliminary clinical activity of a novel pancreatic enzyme preparation in pancreatic insufficient cystic fibrosis patients. Pancreas 2006;32:258-263.

20 Strandvik B, Einarsson K, Lindblad A, Angelin B: Bile acid kinetics and biliary lipid composition in cystic fibrosis. J Hepatol 1996;25: $43-48$.
21 Harries JT, Muller DPR, McCollum JPK, et al: Intestinal bile salts in cystic fibrosis. Arch Dis Child 1979;54:19-24.

22 Perin N, Jarocka-Cyrta E, Keelan M, et al: Dietary lipid composition modifies intestinal morphology and nutrient transport in young rats. J Pediatr Gastroenterol Nutr 1999;28:46-53.

23 Maqbool A, Schall J, Zemel B, et al: Essential fatty acid deficiency and the relevance of the triene:tetraene ratio in children with cystic fibrosis. J Pediatr Gastroenterol Nutr 2005; 41:550.

24 Strandvik B, Berg U, Kallner A, Kusoffsky E: Effect on renal function of essential fatty acid supplementation in cystic fibrosis. J Pediatr 1989;115:242-250.

25 Strandvik B, Hultcrantz R: Liver function and morphology during long term fatty acid supplementation in cystic fibrosis. Liver 1994;14:32-36.

26 van Biervliet S, De Waele K, van Winckel M, Robberecht E: Percutaneous endoscopic gastrostomy in cystic fibrosis: patient acceptance and effect of overnight tube feeding on nutritional status. Acta Gastroenterol Belg 2004;67:241-244.

27 Brown RK, Kelly FJ: Evidence for increased oxidative damage in patients with cystic fibrosis. Pediatr Res 1994;36:487-493.

28 Sinaasappel M, Stern M, Littlewood J, et al: Nutrition in patients with cystic fibrosis: a European Consensus. J Cyst Fibr 2002;1:5175.

29 Borowitz D, Baker RD, Stallings V: Consensus report on nutrition for pediatric patients with cystic fibrosis. J Pediatr Gastroenterol Nutr 2002;35:246-259.

30 Proesmans M, De Boeck K: Evaluation of dietary fiber intake in Belgian children with cystic fibrosis: Is there a link with gastrointestinal complaints? J Pediatr Gastroenterol Nutr 2002;35:610-614

31 Ferkol T, Rosenfeld M, Milla CE: Cystic fibrosis pulmonary exacerbations. J Pediatr 2006;148:259-264.

32 Hollsing AE, Lantz B, Bergström $\mathrm{K}$, et al: Granulocyte elastase-alpha1-antiproteinase complex in cystic fibrosis: sensitive plasma assay for monitoring pulmonary infections. J Pediatr 1987;111:206-211.

33 Strandvik B: Antibiotic therapy of pulmonary infections in cystic fibrosis. Dosage and dose schedules. Chest 1988;94:146S-149S.

34 Koch C, Frederiksen B, Hoiby N: Patient cohorting and infection control. Semin Respir Crit Care Med 2003;24:703-716.

35 Steinkamp G, Ullrich G: Different opinions of physicians on the importance of measures to prevent acquisition of Pseudomonas aeruginosa from the environment. J Cyst Fibros 2003;2:199-205.
36 Wood DM, Smyth AR: Antibiotic strategies for eradicating Pseudomonas aeruginosa in people with cystic fibrosis. The Cochrane Database of Systematic Reviews 2006; DOI: 10.1002/14651858.CD004197.pub2.

37 Strandvik B, Hjelte L, Malmborg AS: Cystic fibrosis pulmonary infection: the Swedish experience; in Bauernfeind A, Marks MI, Strandvik B (eds): Cystic Fibrosis Pulmonary Infections: Lessons from Around the World. Basel, Birkhäuser, 1996, pp 293-302.

38 Blomquist M, Freyschuss U, Wiman LG, Strandvik B: Physical activity and self treatment in cystic fibrosis. Arch Dis Child 1986; 61:362-367.

39 Suri R: The use of human deoxyribonuclease (rhDNase) in the management of cystic fibrosis. BioDrugs 2005; 19:135-144.

40 Elkins MR, Robinson M, Rose BR, et al: A controlled trial of long-term inhaled hypertonic saline in patients with cystic fibrosis. $\mathrm{N}$ Engl J Med 2006;354:229-240.

41 Hollsing AE, Granström M, Strandvik B: Prospective study of serum staphyloccocal antibodies in cystic fibrosis. Arch Dis Child 1987;62:905-911.

42 Cantón R, Cobos N, de Gracia J, et al; on behalf of the Spanish Consensus Group for Antimicrobial Therapy in the Cystic Fibrosis Patient: Antimicrobial therapy for pulmonary pathogenic colonisation and infection by Pseudomonas aeruginosa in cystic fibrosis patients. Clin Microbiol Infect 2005;11:690-703.

43 Ericsson-Hollsing A, Granström M, Vasil M, et al: Prospective study of serum antibodies to Pseudomonas aeruginosa exoproteins in cystic fibrosis. J Clin Microbiol 1987;25: 1868-1874.

44 Cheer SM, Waugh J, Noble S: Inhaled tobramycin (TOBI): a review of its use in the management of Pseudomonas aeruginosa infections in patients with cystic fibrosis. Drugs 2003;63:2501-2520.

45 Blumer JL, Saiman L, Konstan MW, Melnick $\mathrm{D}$ : The efficacy and safety of meropenem and tobramycin vs ceftazidime and tobramycin in the treatment of acute pulmonary exacerbations in patients with cystic fibrosis. Chest 2005; 128:2336-2346.

46 Strandvik B, Hjelte L, Malmborg AS, Widén B: Home intravenous antibiotic treatment of patients with cystic fibrosis. Acta Paediatr Scand 1992;81:340-344.

47 Parmar JS, Nasser S: Antibiotic allergy in cystic fibrosis. Thorax 2005;60:517-520.

48 Dezateux C, Walters S, Balfour-Lynn I: Inhaled corticosteroids for cystic fibrosis. The Cochrane Database Systematic Reviews 2000;2:CD001915.

49 Villa MP, Pagani J, Lucidi V, et al: Nocturnal oximetry in infants with cystic fibrosis. Arch Dis Child 2001;84:50-54.

50 Mallory GB, Fullmer JJ, Vaughan DJ: Oxygen therapy for cystic fibrosis. The Cochrane Database of Systematic Reviews 2005;4. DOI: 10.002/14651858. CD003884.pub2. 
51 Dobbin CJ, Milross MA, Piper AJ, et al: Sequential use of oxygen and bi-level ventilation for respiratory failure in cystic fibrosis. J Cyst Fibros 2004;3:237-242.

52 Rosenbluth DB, Wilson K, Ferkol T, Schuster DP: Lung function decline in cystic fibrosis patients and timing for lung transplantation referral. Chest 2004;126:412-419

53 Grosse SD, Boyle CA, Botkin JR, et al: CDC: Newborn screening for cystic fibrosis: evalutation of benefits and risks and recommendations for state newborn screening programs. MMWR Recomm Rep 2004;53:136.
54 Dankert-Roelse JE, Mérelle ME: Review of outcomes of neonatal screening for cystic fibrosis versus non-screening in Europe. J Pediatr 2005; 147:S15-S20.

55 Castellani C: Evidence for newborn screening for cystic fibrosis. Paediatr Respir Rev 2003;4:278-284.

56 Lannefors L, Lindgren A: Demographic transition of the Swedish cystic fibrosis community - results of modern care. Respir Med 2002;96:681-685.

57 Meyburg J, Hoffmann GF: Liver transplantation for inborn errors of metabolism. Transplantation 2005;80:S135-S137.
58 Fridell JA, Vianna R, Kwo PY, et al: Simultaneous liver and pancreas transplantation in patients with cystic fibrosis. Transplant Proc 2005;37:3567-3569.

59 Bech B, Pressler T, Iversen M, et al: Longterm outcome of lung transplantation for cystic fibrosis - Danish results. Eur J Cardiol Thorac Surg 2004;26:1180-1186.

60 Ganesh JS, Rogers CA, Bonser RS, Banner NR: Outcome of heart-lung and bilateral sequential lung transplantation for cystic fibrosis: a UK national study. Eur Respir J 2005;25:964-969. 